\title{
Nonlinear consolidation theory for prefabricated vertical drains with elliptic cylindrical assumption
}

\author{
Huang Chao-Xuan ${ }^{1, *}$, Hu Guo-jie ${ }^{2}$, Li Jun-jie ${ }^{1}$ \\ ${ }^{1}$ Zhejiang Design Institute of Water Conservancy and Hydroelectric Power, Hangzhou 310002, China; \\ ${ }^{2}$ Northwest Institute of Eco-Environment and Resources, Chinese Academy of Sciences, Lanzhou 730000, China;
}

\begin{abstract}
Based on the influence of the non-linear change in the consolidation process of permeability coefficient and compressibility of soft soils and the theory of elliptic cylinders, derived the control equation of the nonlinear consolidation of the vertical foundation of the strip plastic drainage board. Further, according to the complete orthogonal space theory, the equivalent polynomial analytical expression of the nonlinear term is given. Then, the analytic solutions of the nonlinear consolidation are given, and through numerical calculation and verification analysis, it is considered that the Indrarantna (2005) approximation has a relatively small error at $C_{\mathrm{c}} / C_{\mathrm{k}}$ (close to 1 ) and $N_{\mathrm{u}}(<4)$, but when $C_{\mathrm{c}} / C_{\mathrm{k}}$ (difference from 1 ) and $N_{\mathrm{u}}$ are large ( $>4)$, the error is large.
\end{abstract}

\section{Introduction}

Based on the assumption that soil permeability and compressibility in the consolidation process are constant, Terzaghi [1] proposed a classical one-dimensional consolidation theory. However, the consolidation behavior of soft soil is complicated. Therefore, a series of researchers studied nonlinear consolidation theory. Davis \& Raymond [2] firstly got an analytical solution for one-dimensional consolidation problem under instantaneous loading based on the assumption that the permeability coefficient $\mathrm{kv}$ and the volume compression coefficient $\mathrm{mv}$ changed synchronously. Soon after, Barden et al.[3] and Gibson [4] solved one-dimensional nonlinear consolidation theory with numerical methods based on different assumptions on the nonlinearity of soil unit. Mesri et al.[5] studied the one-dimensional large-strain nonlinear consolidation theory based on a series of simplification. Since then, many scholars have conducted in-depth researches from various aspects such as numerical analysis, laboratory tests and field tests. Lekha et.al [6] used approximate method solved a onedimensional nonlinear consolidation.

As for the nonlinear consolidation of vertical drained foundation, Hansbo et.al [7] considered the non-linearity of the permeability coefficient in the consolidation process, analyzed exponential relationship between hydraulic gradient and penetration velocity. Indrarantna et al. [8] used the relationship between e $\log \sigma$, and e $\sim \log k_{\mathrm{v}}$ to determine the relationship between the compression coefficient $C_{\mathrm{c}}$ and the horizontal permeability coefficient $\mathrm{kh}$, and then got an analytical solution for the consolidation of vertical drained ground.

Considering the real size of plastic vertical drain, Huang et al. [9] gained an analytical solution for the consolidation of vertical drained ground with a assumption that the PVD shape is a elliptic cylinder instead of cylinder. Lu et al. [10] analyzed the nonlinear consolidation of vertical foundations with the approximate method.

Thereby, a nonlinear consolidation controlling equation based the elliptic cylinder assumption for vertical drain considering the non-linear change of permeability coefficient and compressibility of soft soil during the consolidation process is derived. Legendre polynomial space theory is applied in the following to solve this equation.

\section{Description of physical model and assumptions}

Because of the advantages of fast construction, good permeability and low cost, prefabricated vertical drain is most widely used among different drain materials in soft soil ground improvement. Fig. 1 is the equivalent calculation model for the elliptical cylinder of PVD ${ }^{[9]}$. In the figure, $L$ is the calculation length of the drain; $\rho_{\mathrm{w}}$ is the length coordinates of the elliptic cylinder drain; $\rho_{\mathrm{s}}$ is the length coordinates of the confocal elliptic cylinder smear zone; $\rho_{\mathrm{e}}$ is the length coordinates of the confocal elliptic influence area of the drainage body; $u$ is the excess pore water pressure in soil; $k_{\mathrm{w}}$ is the permeability coefficient of drain; $k_{\mathrm{h}}$ is the radial permeability coefficient of soil; $k_{\mathrm{s}}$ is the permeability coefficient of smear zone soil.

The basic assumptions are as follows:

(1) Equal strain assumption, the vertical deformation of the same depth between drain and surrounding soil layer are equal.

\footnotetext{
* Corresponding author: hcx8311@mail.zdwp.net
} 
(2) Only the normal flow in the ellipse unit is considered; the seepage in soil is consistent with Darcy's law; well resistance is ignored in drain well.

(3) The permeability coefficient of soil in the smear zone is changed synchronously with the permeability coefficient of the original soil area, $\mathrm{ks} / \mathrm{kh}$ is a fixed value.

(4) The top surface is permeable, while the bottom surface and the periphery of the vertical drain foundation is impervious to water.

(5) The consolidation by radial flow and vertical flow can be considered separately according to Carrilo's law.

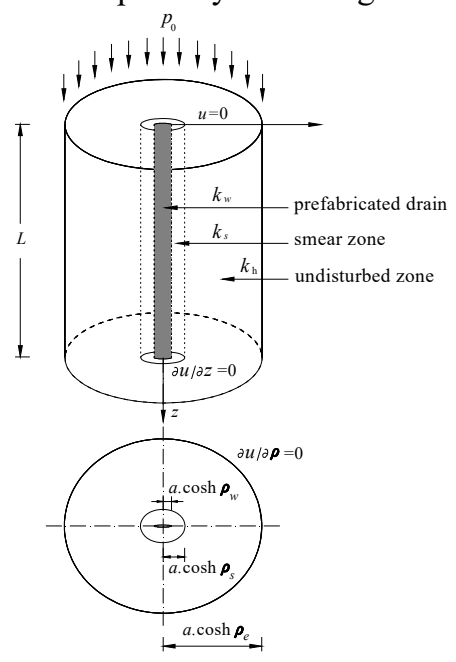

(a) Calculation model

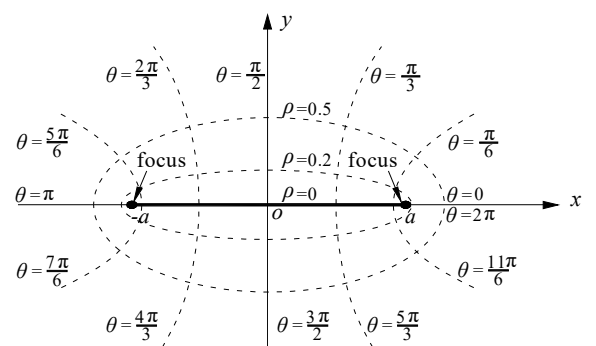

(b) Elliptical coordinate system in $\rho$ and $\theta$ coordinates Fig.1. Diagram of unit cell with elliptic cylinder assumption on PVD

\section{Basic equations and solution conditions}

\subsection{Constitutive relations}

According to Mikasa ${ }^{[5]}$, the relationship between the void ratio $e$, permeability coefficient $k_{\mathrm{h}}$ and effective stress $\sigma^{\prime}$ is:

$$
\begin{aligned}
& e=e_{0}-C_{c} \lg \left(\frac{\sigma^{\prime}}{\sigma_{0}{ }^{\prime}}\right) \\
& e=e_{0}-C_{k} \lg \left(\frac{k_{h}}{k_{h 0}}\right)
\end{aligned}
$$

where $C_{\mathrm{c}}$ and $C_{\mathrm{k}}$ are the compression index and penetration index of soil, respectively; $e_{0}$ and $\sigma_{0}$ ' are the initial void ratio and initial effective stress; $k_{\mathrm{h} 0}$ is the initial permeability coefficient.
According to the effective stress principle, there is

$$
\sigma^{\prime}=\sigma_{0}{ }^{\prime}+p_{0}-\bar{u}_{\rho}
$$

Assuming the distribution of additional stress is constant along the depth direction, then

$$
\frac{m_{v 0}}{m_{v}}=1+\frac{p_{0}}{\sigma_{0}{ }^{\prime}}-R_{u} \cdot \frac{p_{0}}{\sigma_{0}{ }^{\prime}}
$$

where $\bar{u}_{\rho}$ is the pore water pressure; $R_{u}=\bar{u}_{\rho} / p_{0}$.

Then it can be obtained that

$$
\frac{k_{h(t)}}{k_{h 0}}=\left(1+\frac{p_{0}}{\sigma_{0}{ }^{\prime}}-R_{u} \cdot \frac{p_{0}}{\sigma_{0}{ }^{\prime}}\right)^{-C_{c} / C_{k}}
$$

\subsection{Balance equation and continuous equation}

According to the theory of elliptical cylindrical coordinate system (Fig. 1), the basic foundation consolidation equation of the elliptic cylinder drainage body is obtained.

Balance equation:

$$
\frac{\partial \varepsilon_{v}}{\partial t}=-m_{v} \frac{\partial \overline{u_{\rho}}}{\partial t}
$$

Continuous equations for the soil in smear zone and undisturbed area:

$$
\left\{\begin{array}{l}
\frac{k_{s}}{\gamma_{w}} \frac{1}{a^{2}\left(\cosh ^{2} \rho-\cos ^{2} \theta\right)}\left(\frac{\partial^{2} u}{\partial \rho^{2}}+\frac{\partial^{2} u}{\partial \theta^{2}}\right)=\frac{\partial \varepsilon_{v}}{\partial t}, \quad \rho_{w} \leq \rho \leq \rho_{s} \\
\frac{k_{h}}{\gamma_{w}} \frac{1}{a^{2}\left(\cosh ^{2} \rho-\cos ^{2} \theta\right)}\left(\frac{\partial^{2} u}{\partial \rho^{2}}+\frac{\partial^{2} u}{\partial \theta^{2}}\right)=\frac{\partial \varepsilon_{v}}{\partial t}, \quad \rho_{s} \leq \rho \leq \rho_{e}
\end{array}\right.
$$

where $m_{\mathrm{v}}$ is the volume compression coefficient; $\varepsilon_{\mathrm{v}}$ is the volume strain; $u$ is the excess pore-water pressure; $\gamma_{\mathrm{w}}$ is the water bulk density; $t$ is the consolidation time.

The above equation (6) and (7) are the basic consolidation equations in the elliptical cylindrical coordinate system.

\subsection{Controlling equations}

From the assumption of equal strain, $\varepsilon_{\mathrm{V}}$ is independent of $\rho$, and according to the biaxial symmetry of the ellipse and the excess pore pressure solution, it can be expressed as $u=f_{(\rho)}+g_{(\rho)} \cos 2 \theta$. Substituting it into equation (7), then it can be obtained that

$$
\left(\frac{\partial^{2} u}{\partial \rho^{2}}+\frac{\partial^{2} u}{\partial \theta^{2}}\right)=\frac{1}{2}\left(\frac{\gamma_{w} a^{2}}{k_{s}} \frac{\partial \varepsilon_{v}}{\partial t}\right)(\cosh 2 \rho+\cos 2 \theta)
$$

This ordinary differential equation can be solved by separating variables method as:

$$
\left\{\begin{array}{l}
f_{(\rho)} "=\frac{1}{2}\left(\frac{\gamma_{w} a^{2}}{k_{s}} \frac{\partial \varepsilon_{v}}{\partial t}\right) \cosh 2 \rho \\
g_{(\rho)} "-4 g_{(\rho)}=\frac{1}{2}\left(\frac{\gamma_{w} a^{2}}{k_{s}} \frac{\partial \varepsilon_{v}}{\partial t}\right)
\end{array}\right.
$$

According to the boundary conditions: $\left.\frac{\partial f}{\partial \rho}\right|_{\rho=\rho_{e}}=0$ 、 $\left.\frac{\partial g}{\partial \rho}\right|_{\rho=\rho_{e}}=0,\left.g\right|_{\rho=\rho_{w}}=0$, and continuous equations, there are: $\left.f_{w s}\right|_{\rho=\rho_{s}}=\left.f_{s e}\right|_{\rho=\rho_{s}},\left.g_{w s}\right|_{\rho=\rho_{s}}=\left.g_{s e}\right|_{\rho=\rho_{s}}$, the excess pore water pressure in the smear zone and the nature soil area can be solved, as shown in Huang et al. ${ }^{[9]}$. 
According to the principle of integral average, the average excess pore pressure at any depth in the foundation can be expressed. Then it can be calculated as follows

$$
\bar{u}_{\rho}=\frac{\gamma_{w}\left(\cosh \rho_{e}\right)^{2}}{2 k_{h(t)}} a^{2} F_{h} \times\left(\frac{\partial \varepsilon_{v}}{\partial t}\right)
$$

Equation (10) is the deduced controlling equation that ignored well resistance of PVD with elliptic cylindrical assumption. Parameter $F_{\mathrm{h}}$ can be calculated by the basic parameters $\rho_{\mathrm{w}}, \rho_{\mathrm{s}}, \rho_{\mathrm{e}}, a$, and $k_{\mathrm{h}} / k_{\mathrm{s}}$. The calculation process is attached in Huang et al. ${ }^{[9]}$.

Substituting equation (5)、 (6) into equation (10), and combining $R_{u}=\frac{\bar{u}_{\rho}}{p_{0}}$, it can be obtained that

$$
R_{u}=-\frac{\gamma_{w} a^{2}\left(\cosh \rho_{e}\right)^{2}}{2} \frac{m_{v 0}}{k_{h 0}} F_{h} \times\left(1+\frac{p_{0}}{\sigma_{0}{ }^{\prime}}-R_{u} \cdot \frac{p_{0}}{\sigma_{0}{ }^{\prime}}\right)^{C_{c} / C_{k}-1} \frac{\partial R_{u}}{\partial t}
$$

Assume a dimensionless time parameter : $T=\frac{c_{h 0}}{4 a^{2}\left(\cosh \rho_{e}\right)^{2}} t^{\prime} \quad N_{u}=\frac{p_{0}}{\sigma_{0}{ }^{\prime}}$, and the above equation (11) can be simplified as a dimensionless equation as

$$
\frac{\partial R_{u}}{\partial T}=-\frac{8}{F_{h}}\left(1+N_{u}-R_{u} \cdot N_{u}\right)^{1-C_{c} / C_{k}} \cdot R_{u}
$$

where

$$
c_{h 0}=\frac{k_{h 0}\left(1+e_{0}\right)}{\gamma_{w}\left(\frac{\partial e}{\partial \sigma^{\prime}}\right)_{t=0}} m_{v 0}=\left(\frac{\partial e}{\partial \sigma^{\prime}}\right)_{t=0} \cdot \frac{1}{1+e_{0}} .
$$

The above equation (12) is the basic equation for the nonlinear consolidation of the PVD foundation in the elliptical cylindrical coordinate system without considering the well resistance.

\section{Nonlinear equation solving}

According to the nonlinear control equation (12) can be transformed into:

$$
-\frac{8}{F_{h}} \partial T=\frac{\left(1+N_{u}-R_{u} \cdot N_{u}\right)^{C_{c} / C_{k}-1}}{R_{u}} \partial R_{u}
$$

In this paper, the rigorous analytical solution of equation (13) is given based on Hillbert's complete orthogonal space theory in functional analysis.Let $C_{c} / C_{k}=\left\langle C_{c} / C_{k}\right\rangle+M_{c k}$, and $\left\langle C_{c} / C_{k}\right\rangle, M_{c k}$ are functions of $C_{c} / C_{k}$, and their values are as follows:

while $0<C_{c} / C_{k} \leq 2,\left\{\begin{array}{l}\left\langle C_{c} / C_{k}\right\rangle=C_{c} / C_{k} ; \text { while } \\ M_{c k}=0\end{array}\right.$

$$
2<C_{c} / C_{k} \leq 3,\left\{\begin{array}{l}
\left\langle C_{c} / C_{k}\right\rangle=C_{c} / C_{k}-1 ; \\
M_{c k}=1
\end{array}\right.
$$

In turn, nonlinear equation (13) can be transformed into:

$$
-\frac{8}{F_{h}} \partial T=\left(1+N_{u}-R_{u} \cdot N_{u}\right)^{\left\langle C_{c} / C_{k}\right\rangle-1} \times \frac{\left(1+N_{u}-R_{u} \cdot N_{u}\right)^{M_{c k}}}{R_{u}} \partial R_{u}
$$

The non-linear item on the right side of (19) is: $G\left(R_{u}\right)=\left(1+N_{u}-R_{u} \cdot N_{u}\right)^{\left\langle C_{c} / C_{k}\right\rangle-1}$, when $0<R_{\mathrm{u}}<1$, The function $G\left(R_{\mathrm{u}}\right)$ about $R_{\mathrm{u}}$ is extended from the interval
$[0,1]$ to the even function in the complete Legendre polynomial space $[-1,1]$.

According to Hillbert orthogonal complete space theory, the Negejronde polynomial orthogonal can be represented by the Legendre polynomial equivalent:

$$
G\left(R_{u}\right)=\left(1+N_{u}-R_{u} \cdot N_{u}\right)^{\left\langle C_{c} / C_{k}\right\rangle-1}=a_{0} \cdot \mathrm{P}_{0}(x)+a_{2} \cdot \mathrm{P}_{2}(x)+a_{4} \cdot \mathrm{P}_{4}(x)+\cdots
$$

Where $\mathrm{P}_{0}(\mathrm{x}), \mathrm{P}_{2}(\mathrm{x})$, and $\mathrm{P}_{4}(\mathrm{x})$ represent Legendre polynomials, respectively, $\mathrm{P}_{0}(x)=1$ 、 $\mathrm{P}_{2}(x)=\frac{1}{2}\left(3 x^{2}-1\right)$ 、 $\mathrm{P}_{4}(x)=\frac{1}{8}\left(35 x^{4}-30 x^{2}+3\right) \cdot$

Then equation (14) can in turn be transformed into: $-\frac{8}{F_{h}} \partial T=\frac{a_{0} \cdot \mathrm{P}_{0}(x)+a_{2} \cdot \mathrm{P}_{2}(x)+a_{4} \cdot \mathrm{P}_{4}(x)}{R_{u}} \cdot\left(1+N_{u}-R_{u} \cdot N_{u}\right)^{M_{c k}} \partial R_{u}$

According to the orthogonal inner product relationship, it can be seen that the formulas $a_{0}, a_{2}, a_{4}$ can be given as:

$$
\begin{gathered}
a_{0}=\frac{\int_{0}^{1} G(x) \times P_{0}(x) \mathrm{d} x}{\int_{0}^{1} P_{0}(x) \times P_{0}(x) \mathrm{d} x}, \\
a_{2}=\frac{\int_{0}^{1} G(x) \times P_{2}(x) \mathrm{d} x}{\int_{0}^{1} P_{2}(x) \times P_{2}(x) \mathrm{d} x}, \quad a_{4}=\frac{\int_{0}^{1} G(x) \times P_{4}(x) \mathrm{d} x}{\int_{0}^{1} P_{4}(x) \times P_{4}(x) \mathrm{d} x}
\end{gathered}
$$

Then we can give the analytical expressions of the parameters $a_{0}, a_{2}, \quad a_{4}$ about the constant parameters $N_{u}=\frac{p_{0}}{\sigma_{0}{ }^{\prime}}, C_{c} / C_{k}$ :

$$
\begin{aligned}
& a_{0}=\frac{y_{0}}{N_{u}}, \\
& a_{2}=\frac{30}{4 N_{u}^{3}}\left\{y_{2}-2\left(1+N_{u}\right) \cdot y_{1}+\left(1+N_{u}\right)^{2} \cdot y_{0}\right\}-\frac{10}{4 N_{u}} y_{0}, \\
& a_{4}=\frac{9}{8}\left\{\frac{30}{N_{u}^{3}}\left[y_{2}-2\left(1+N_{u}\right) y_{1}+\left(1+N_{u}\right)^{2} y_{0}\right]+\frac{3}{N_{u}} y_{0}\right\} \\
& +\frac{315}{8 N_{u}^{5}}\left[y_{4}-4\left(1+N_{u}\right) y_{3}+6\left(1+N_{u}\right)^{2} y_{2}-4\left(1+N_{u}\right)^{3} y_{1}+\left(1+N_{u}\right)^{4} y_{0}\right]
\end{aligned}
$$

where:

$$
\begin{aligned}
& y_{0}=\frac{\left(1+N_{u}\right)^{\left\langle c_{c} / c_{k}\right\rangle}-1}{\left\langle c_{c} / c_{k}\right\rangle} ; \quad y_{1}=\frac{\left(1+N_{u}\right)^{\left\langle c_{c} / c_{k}\right\rangle+1}-1}{\left\langle c_{c} / c_{k}\right\rangle+1} ; \\
& y_{2}=\frac{\left(1+N_{u}\right)^{\left\langle c_{c} / c_{k}\right\rangle+2}-1}{\left\langle c_{c} / c_{k}\right\rangle+2} ; y_{3}=\frac{\left(1+N_{u}\right)^{\left\langle c_{c} / c_{k}\right\rangle+3}-1}{\left\langle c_{c} / c_{k}\right\rangle+3} \\
& y_{4}=\frac{\left(1+N_{u}\right)^{\left\langle c_{c} / c_{k}\right\rangle+4}-1}{\left\langle c_{c} / c_{k}\right\rangle+4}
\end{aligned}
$$

The Legendre polynomials $P_{0}(x), P_{2}(x)$, and $P_{4}(x)$ are substituted into equation (16). The following simple equations can be obtained by simple changes:

$$
\begin{gathered}
-\frac{8}{F_{h}} \partial T=\left(b_{0}+b_{2} R_{u}{ }^{2}+b_{4} R_{u}{ }^{4}\right) \times \frac{\left(1+N_{u}-R_{u} \cdot N_{u}\right)^{M_{c k}}}{R_{u}} \partial R_{u}(20) \\
\text { where : } \quad b_{0}=a_{0}-\frac{1}{2} a_{2}+\frac{3}{8} a_{4} ; \quad b_{2}=\frac{3}{2} a_{2}-\frac{30}{8} a_{4} ;
\end{gathered}
$$
$b_{4}=\frac{35}{8} a_{4}^{\circ}$ 
According to equation (20) deduced in this paper, we can give an analytical solution, as follows

1) while $0<C_{c} / C_{k} \leq 2,\left\{\begin{array}{l}\left\langle C_{c} / C_{k}\right\rangle=C_{c} / C_{k} \\ M_{c k}=0\end{array}\right.$, according to the separation integral, and the boundary conditions $T=0$ and $R_{u}=1$,

$$
T=-\frac{F_{h}}{8}\left\{b_{0} \ln R_{u}+\frac{b_{2}}{2}\left(R_{u}{ }^{2}-1\right)+\frac{b_{4}}{4}\left(R_{u}{ }^{4}-1\right)\right\}
$$

2) while $2<C_{c} / C_{k} \leq 3$, $\left\{\left\langle C_{c} / C_{k}\right\rangle=C_{c} / C_{k}-1\right.$, according to the boundary $M_{c k}=1$

conditions $T=0$ and $R_{u}=1$,

$$
T=-\frac{F_{h}}{8}\left\{\begin{array}{l}
\left(1+N_{u}\right) b_{0} \ln R_{u}-N_{u} b_{0}\left(R_{u}-1\right)+\left(1+N_{u}\right) \frac{b_{2}}{2}\left(R_{u}{ }^{2}-1\right) \\
-N_{u} \frac{b_{2}}{3}\left(R_{u}{ }^{3}-1\right)+\left(1+N_{u}\right) \frac{b_{4}}{4}\left(R_{u}{ }^{4}-1\right)-N_{u} \frac{b_{4}}{5}\left(R_{u}{ }^{5}-1\right)
\end{array}\right\}
$$

Among them, total average degree of consolidation $U=1-R_{\mathrm{u}}$. So far, the equivalent analytical solution of the nonlinear consolidation of shaft foundations has been given, that is, the relationship between the degree of consolidation, the dimensionless time factor $T$, and the analytical solution.

\section{Verification and discussion}

\subsection{Verification}

In order to compare and analyze the relationship between the nonlinear consolidation analytical solution (22) in this paper and the nonlinear consolidation approximate solution in B Indraratna (2005) under ideal shaft conditions, this paper compares and analyzes the following calculation conditions:

Condition（a）: $C_{\mathrm{c}} / C_{\mathrm{k}}=1, N_{\mathrm{u}}=20, F_{\mathrm{h}}=8$;

Condition（b）: $C_{\mathrm{c}} / C_{\mathrm{k}}=0.2, N_{\mathrm{u}}=10, F_{\mathrm{h}}=8$;

Condition (c) : $C_{\mathrm{c}} / C_{\mathrm{k}}=0.2, N_{\mathrm{u}}=20, F_{\mathrm{h}}=8$;

Condition (d): $C_{\mathrm{c}} / C_{\mathrm{k}}=1.95, N_{\mathrm{u}}=10, F_{\mathrm{h}}=8$;

Condition (e) : $C_{\mathrm{c}} / C_{\mathrm{k}}=1.95, N_{\mathrm{u}}=20, F_{\mathrm{h}}=8$;

Condition（f）: $C_{\mathrm{c}} / C_{\mathrm{k}}=2.95, N_{\mathrm{u}}=20, F_{\mathrm{h}}=8$;

It can be seen from Fig. 2 that when $C_{\mathrm{c}} / C_{\mathrm{k}} \rightarrow 1$, the analytical solution of nonlinear consolidation in this paper degenerates to classic linear consolidation. At this time, the analytical solution in this paper is consistent with the calculation results of Indraratna et al. ${ }^{[8]}$ approximation method, and is consistent with the accurate numerical solutions.

\subsection{Discussion}

Through the comparison of numerical calculations under the above different conditions:

(1) The analytical solution in this paper degenerates into an analytical solution similar to an ideal shaft when $c_{\mathrm{c}} / c_{\mathrm{k}}$ approaches1, which is consistent with the Indrarantna $^{[8]}$ approximation, but without the dimensionless parameters $F_{\mathrm{h}}$, the main reason is that the difference between the shape of the strip-shaped plastic drainage plate and the circular sand well is larger.

(2) The larger the relative value of the pre-loading force $N_{\mathrm{u}}$ is $\left(N_{\mathrm{u}}=10\right)$, the deviation of Indrarantna ${ }^{[8]}$ approximation and the exact numerical solution is larger even the degree of consolidation error as high as $30 \%$ when $C_{\mathrm{c}} / C_{\mathrm{k}}$ deviates from 1 and gradually approaches 0.2 , The results of the special Indrarantna ${ }^{[8]}$ approximation seriously overestimate the nonlinear consolidation rate of shaft foundations when the degree of consolidation is less than $90 \%$. In contrast, the results of the analytical solution in this paper are basically the same to the exact numerical solution.

(3) The larger the relative value of the preloading force $N_{\mathrm{u}}$ is $\left(N_{\mathrm{u}}=10\right)$, the deviation of Indrarantna ${ }^{[8]}$ approximation and the exact numerical solution is larger even the degree of consolidation error as high as $40 \%$ when $C_{\mathrm{c}} / C_{\mathrm{k}}$ deviates from 1 and gradually approaches 2 . The results of the Indrarantna ${ }^{[8]}$ approximation seriously overestimates the nonlinear consolidation rate of the shaft foundation especially when the degree of consolidation is less than $95 \%$. In contrast, the results of the analytical solution in this paper are basically the same to the exact numerical solution.
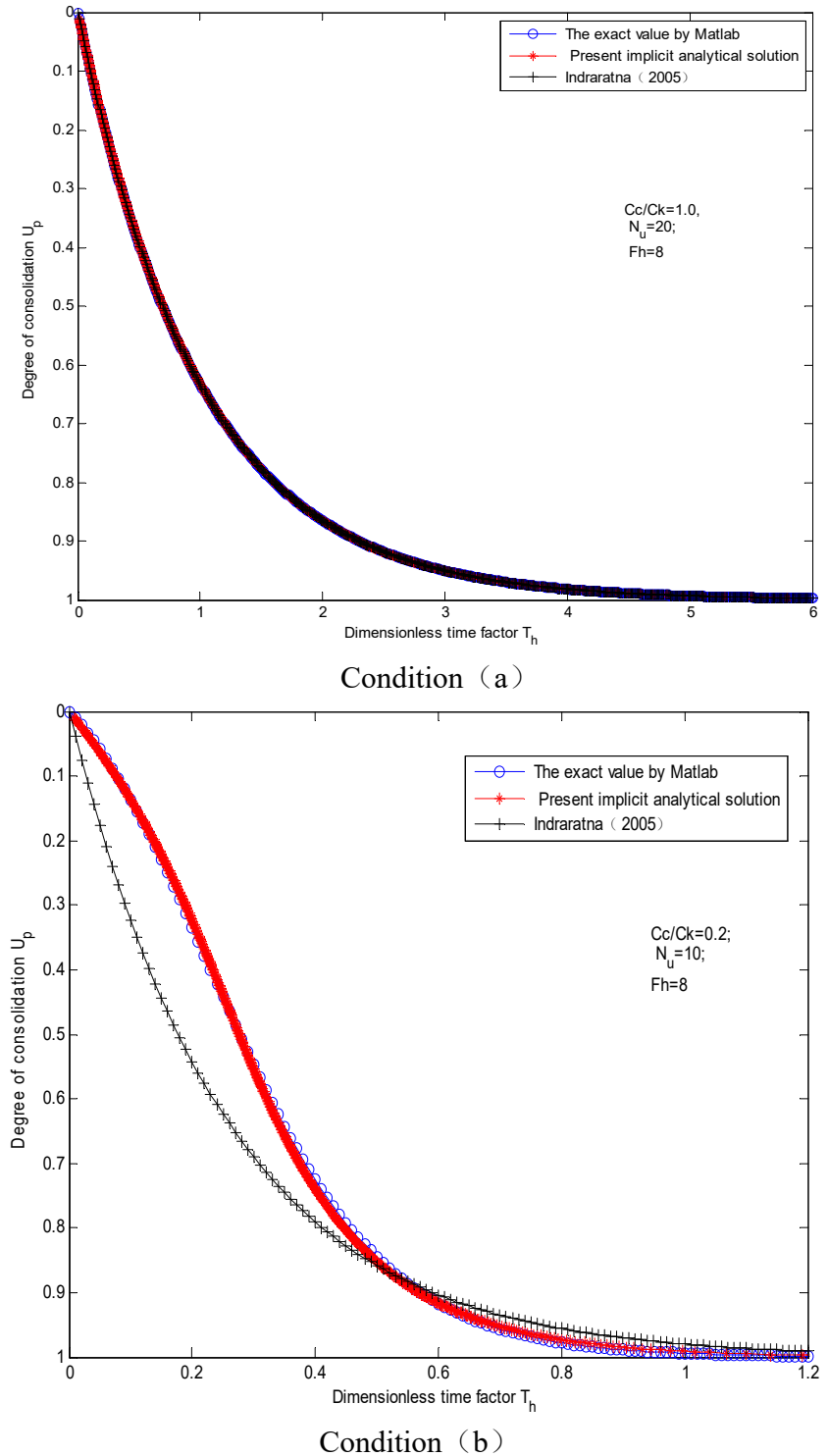

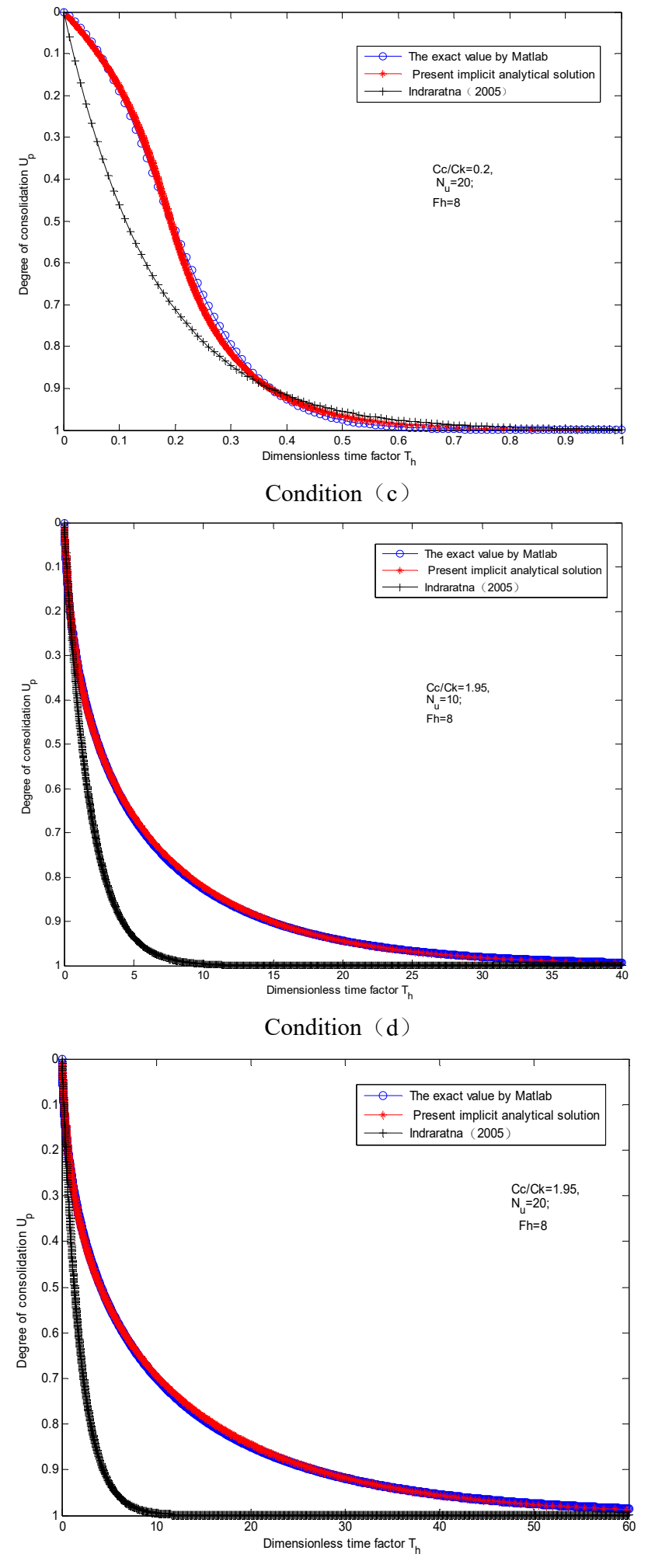

Condition (e)

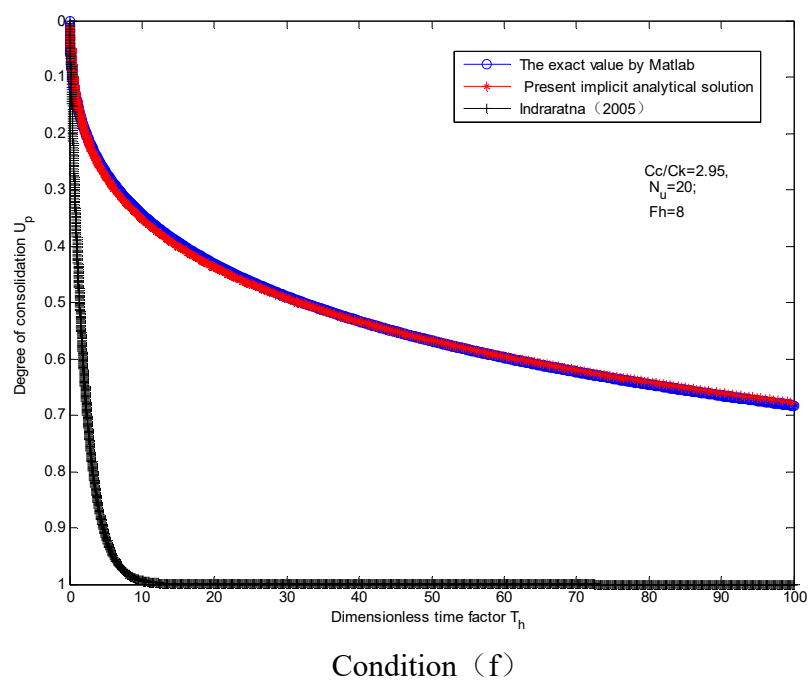

Fig. 2 Comparison of analytical solutions and different solutions

\section{Conclusion}

Firstly, based on the theory of elliptic cylinders, considering the nonlinear influence of permeability coefficient and compressibility of soft soil in the consolidation process, the the nonlinear consolidation of shaft foundation is discussed.

Secondly, derived the control equation of the nonlinear consolidation of the vertical foundation of the strip plastic drainage board. Further, according to the complete orthogonal space theory, the equivalent polynomial analytical expression of the nonlinear term is given. Then, the analytic solution of the nonlinear consolidation degree are given respectively.

Thirdly, through numerical calculation and verification analysis, it is considered that the Indrarantna (2005) approximation has a relatively small error at $\mathrm{C}_{\mathrm{c}} / \mathrm{C}_{\mathrm{k}}$ (close to 1 ) and $N_{\mathrm{u}}(<4)$, but when $\mathrm{C}_{\mathrm{c}} / \mathrm{C}_{\mathrm{k}}$ (difference from 1) and $N_{\mathrm{u}}$ are large ( $\left.>4\right)$, the error is large .

Finally, it is recommended to conduct follow-up indepth research on the nonlinear consolidation of the shaft foundation under the influence of well resistance.

\section{Acknowledgments}

This work was supported by Zhejiang Province Basic Public Welfare Research Project (LGF18E090004), Zhejiang Water Resources Department Science and Technology Project (RC1701).

\section{References}

1. Terzaghi . Theoretical Soil Mechanics[M]. Wiley, New York. 1943.

2. DAVIS E H, RAYMOND G P (1965). A nonlinear theory of consolidation[J]. Geotechnique, 15(2): $161-173$.

3. BARDEN L, BERRY P L(1965). Consolidation of 
normally consolidated clay[J]. Journal of the Soil Mechanics and Foundation Division, ASCE, 91(SM5): 15-35.

4. Gibson, R. E., England, G. L. and Hussey, M. J. L(1967). The theory of one-dimensional consolidation of saturated clays, 1. Finite nonlinear consolidation of thin Homogeneous layers[J]. Geotechnique, 17(3), 261-273.

5. MESRI G, ROKHSAR A (1974). Theory of consolidation for clays[J]. Journal of Geotechnical Engineering, ASCE, 100(8): 889904.

6. Lekha, K.R., Krishnaswamy, N.R. and Basak, P (2003). Consolidation of clays for variable permeability and compressibility[J].Journal of Geotechnical and Geoenvironmental Engineering, ASCE, 129(11):1001-1009.

7. S Hansbo, M Jamiolkowski (1981). Consolidation by vertical drains[J]. Géotechnique, 31 (31) :4566

8. B Indraratna, C Rujikiatkamjorn, I Sathananthan(2005). Radial consolidation of clay using compressibility indices and varying horizontal permeability[J]. Canadian Geotechnical Journal, 42(5): 1330-1341.

9. HUANG C X, DENG Y B (2016). Consolidation theory for prefabricated vertical drains with elliptic cylindrical assumption[J]. Computers and Geotechnics,77(1): 156-166.

10. Mengmeng Lu, Shanyong Wang, Scott W. Sloan, et.al(2015). Nonlinear consolidation of vertical drains with coupled radial-vertical flow considering well resistance [J]. Geotextiles \& Geomembranes , 43 (2) :182-189 